\title{
Node-Based SIRS Model on Heterogeneous Networks: Analysis and Control*
}

\author{
Fangzhou $\mathrm{Liu}^{1}$ and Martin Buss ${ }^{1}$
}

\begin{abstract}
In this paper, a node-base susceptible-infectedrecovered-susceptible (SIRS) model on heterogeneous networks is proposed. The condition for global exponential stability of the origin in the disease-free case is obtained via Lyapunov theory. Furthermore, aiming at regulating the probabilities of being infected and recovered to desired values, the controlled recovering rates are designed. Taking practical implementation into consideration, an alternative approach is provided to calculate the feasible control inputs. Finally, several numerical simulations validate our results.
\end{abstract}

\section{INTRODUCTION}

The whole world is getting more connected than ever before in human history as a result of technological progress. Networks which facilitate this connectivity have become ubiquitous in our daily life. Among all kinds of networks, no matter whether the components are biological cells, computers, vehicles or human beings, information diffusion on networks plays an important role. Thus information diffusion has drawn a lot of attention in recent years [1]-[3].

To describe the diffusion of information, innovation or influence on networks, a multitude of mathematical models have been reported. Generally, they can be classified into macroscopic and microscopic models. Macroscopic models mostly focus on the phenomena of diffusion on a large scale, e.g. the proportion of people who are infected with certain disease or the amount of computers which are attacked by viruses. The typical epidemic models are essential among all macroscopic models, among which SI model is fundamental. By labelling persons as susceptible (S) and infected (I), differential equations can be utilized to model the process of a person turning from state $\mathrm{S}$ to state I because of the influence of other characters in state I. Extensions of the SI model, such as SIS and SIR models are also fully studied [4], [5], especially on the existence of non-zero threshold, e.g. the work in [6] on small-world network.

In contrast to macroscopic models, microscopic models are mainly node-based. They concentrate on the states of every individual in the whole network, e.g. the possibility of an individual adopting certain innovation. The Linear threshold (LT) model [7] describes the way in which a

\footnotetext{
*The research leading to these results has received funding partly from the European Research Council under the European Unions Seventh Framework Programme (FP/2007-2013) / ERC Grant Agreement no. 26787, Project SHRINE and partly from the Technische Universität München - Institute for Advanced Study (www.tum-ias.de), funded by the German Excellence Initiative.

${ }^{1}$ Fangzhou Liu and Martin Buss are with the Chair of Automatic Control Engineering and TUM Institute for Advanced Study, Technische Universität München, Lichtenbergstrasse 2a, 85748 Garching, Germany \{fangzhou.liu, mb\}etum.de
}

person updates his decision, i.e. once the weighted sum of her activated neighbors reaches her own threshold, she takes the strategy then. In spite of successful influence of neighbors, the independent cascade model (ICM) is proposed in [8], by introducing the propagation possibility. Apart from that, epidemic models can also be applied microscopically, for instance by taking the probabilities of an individual being susceptible and infected as the state variables. The socalled $N$-intertwined SIS model has been well studied. The remarkable property that there exists an epidemic threshold is given in [9] and the stability of the equilibria in metastable states is proved in [10]. However, no results on node-based SIRS model are reported, which motivates our work in this paper. With the proposed node-based SIRS model, the recovered state is introduced so that the situation of an individual in a network can be more precisely described. From practical point of view, the intermediate state between active and inactive, e.g. ignorance of political propagation, traffic radio news and advertisements of certain product in a period of time, can be modelled as the recovered state, which cannot be covered by existed models.

Another motivation of this paper is the heterogeneity of networks. Generally, a feature of heterogeneous network is the numbers of contacted individuals (neighbors) are heterogeneously distributed. This is the common phenomena in biological, social and transportation networks. Traditional studies, however, focus on homogeneous networks. Kephart and White [11], who are among the earliest researchers, built a SIS model on Erdös-Rényi random graph and all nodes are assumed to have the same degree. Recently, the study of heterogeneous networks receives more attention. In [12], the SI model is applied to campaigning in which not only the different degrees of inspected nodes but also the degrees of the neighbors are considered. In our paper, another part of heterogeneity is taken into consideration. We assume the transition rates of different nodes to be heterogeneous. The heterogeneity that the rates differ for different individuals is a shared feature of real systems. For instance, in social networks, a population often contains a mix of men and women, weak and strong, or outgoing and introverted. This diversity of persons ends up in the heterogeneity of the network. Further examples can also be found in other networks such as data communication networks or transportation networks.

The rest of the paper is organized as follows. Section II contains, as the preliminaries, the detailed introduction of networks, graph theory and the SIRS model. The nodebased SIRS model is established in Section III. Equilibria and 
stability analysis along with control design are presented in Section IV, which are the main results of this paper. Numerical studies are given in Section V. Finally, we conclude this paper in Section VI.

\section{Preliminaries}

\section{A. Networks and Graph Theory}

A network, which is the fundamental medium of information diffusion, is usually described mathematically by a graph whose nodes (vertices) and links (edges) represent the elements of the system and the relations or interactions among them, respectively [13]. Accordingly, the structure of human networks, e.g. social networks, can also be modeled as graphs, where individuals are represented by vertices and the communication is shown by edges. For convenience, the words nodes, vertices, players and agents are used interchangeably in this paper.

Let our problem be described by an undirected connected graph $\mathcal{G}(\mathcal{V}, \mathcal{E})$ with $\mathrm{N}$ nodes, where $\mathcal{V}$ and $\mathcal{E}$ are the sets of nodes and edges, respectively. This yields a symmetric weighted adjacency matrix $W$ of the graph $\mathcal{G}$. For each entry $w_{i j}$ in $W$, the following properties hold: a) $w_{i j} \in\{0,1\}$, b) $w_{i j}=0, i \neq j$ if and only if agent $i$ and agent $j$ are not connected and c) $w_{i i}=0$. The neighborhood $\mathcal{N}$ of node $v_{i}$ is defined as the set of nodes which are connected to it, i.e.

$$
\mathcal{N}\left(v_{i}\right)=\left\{v_{j}: w_{i j}=1, v_{j} \in \mathcal{V}\right\}
$$

Since only the connected nodes are assumed to (be able to) influence each other directly, $\mathcal{N}\left(v_{i}\right)$ is of great importance in this paper. Apart from that, $\mathcal{N}\left(v_{i}\right)$ is not an empty set for each $v_{i}$ because only connected graphs are considered.

\section{B. The SIRS Model}

The Susceptible-infected-recovered-susceptible (SIRS) model, which is an extension of classic SIS and SIR models, has been well studied from the macroscopic point of view [14]. The fundamental SIRS model is presented as follows

$$
\begin{aligned}
\frac{\mathrm{d} S(t)}{\mathrm{d} t} & =-a S(t) I(t)+b R(t), \\
\frac{\mathrm{d} I(t)}{\mathrm{d} t} & =a S(t) I(t)-c I(t), \\
\frac{\mathrm{d} R(t)}{\mathrm{d} t} & =c I(t)-b R(t),
\end{aligned}
$$

where $S, I$ and $R \in[0,1]$ are the proportions of the people who are susceptible, infected and recovered, respectively. The parameters $a, b$ and $c \in \mathbb{R}_{+}$are the related constant transition rates. The SIRS model differs from SIS or SIR model because of the short-term immunity considered in [15]. The above SIRS model can be derived by utilizing Kurtz's theorem while the population is large enough [16]. An intuitive explanation to this model, taking the first equation as an example, is that the population of susceptible can change only because of the influence of the infected population (from $S$ to I) and the number of recovered people who turns to susceptible (from $\mathrm{R}$ to $\mathrm{S}$ ) again. The SIRS model has been extended considering natural birth and death

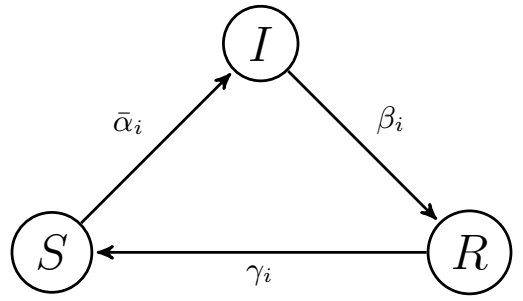

Fig. 1. Node-based SIRS model of node $v_{i}$ with transition rates $\bar{\alpha}_{i}, \beta_{i}$ and $\gamma_{i}$ between different states

and a vaccination process from $S$ to $R$ in [17]. Note that the system above only focuses on the macroscopic behavior of the population, but does not give insight into single character. To describe and analyse the behavior of each individual, we propose a node-based model.

\section{NODE-BASED SIRS MODEL}

In this paper, we study SIRS models focusing on the states of nodes in heterogeneous networks. We assume that there are three possible states in each node, i.e. susceptible $(\mathrm{S})$, infected (I) and recovered (R), which an individual can have when facing a disease spreading in the groups where she belongs to. The disease propagates only via interactions between people. Thus the person we study has no chance of getting infected unless at least one of her neighbors is infected. As is presented in Figure 1, a certain node $v_{i}$, representing a person in a network, can change to different states with different transition rates. All the processes, i.e. the transmission process from $\mathrm{S}$ to $\mathrm{I}$, the recovering process from $\mathrm{I}$ to $\mathrm{R}$ and the process from $\mathrm{R}$ back to $\mathrm{S}$, are all assumed as Poisson processes with positive constant rates, $\bar{\alpha}_{i}, \beta_{i}, \gamma_{i}$, respectively. The transmission rate $\bar{\alpha}_{i}$ of node $v_{i}$ is calculated from mean-field approximation. This process can be explained that node $v_{i}$ gets more possible to be infected with a probability $\alpha_{j} \mathrm{~d} t$ resulting from one connection with an infected neighbor $\left(v_{j}\right)$ in a short time interval $\mathrm{d} t$. Assuming all the influences from neighbors are independent and considering the neighor, e.g. $v_{j} \in \mathcal{N}\left(v_{i}\right)$, is infected with probability $p_{j I}$, the change possibility of the node being infected during $\mathrm{d} t$ is

$$
\left(1-\prod_{j \in \mathcal{N}\left(v_{i}\right)}\left(1-\alpha_{j} p_{j I}\right)\right) \mathrm{d} t .
$$

By omitting the terms with second and higher orders in (1), we obtain

$$
\bar{\alpha}_{i}=\sum_{j=1}^{N} \alpha_{j} \omega_{i j} p_{j I}
$$

which is called the average transmission rate of node $v_{i}$. According to the transformation relations in Figure 1 and the transmission rate in (2), the node-based SIRS model for 
node $v_{i}$ on a heterogeneous network $\mathcal{G}$ is described as

$$
\begin{aligned}
& \dot{p}_{i I}(t)=\left(1-p_{i I}(t)-p_{i R}(t)\right) \sum_{j=1}^{N} \alpha_{j} \omega_{i j} p_{j I}(t)-\beta_{i} p_{i I}(t), \\
& \dot{p}_{i R}(t)=\beta_{i} p_{i I}(t)-\gamma_{i} p_{i R}
\end{aligned}
$$

where $p_{i I}(t) \in[0,1]$ and $p_{i R}(t) \in[0,1]$ are the probabilities of node $v_{i}$ in infected and recovered states at time instance $t$, respectively. Denoting $p_{i S}(t) \in[0,1]$ as the instantaneous probability of node $v_{i}$ being susceptible, it is obvious that the differential equation of $p_{i S}(t)$ is redundant because the node must be in one of the three states, i.e. the equality $p_{i S}(t)+p_{i I}(t)+p_{i R}(t)=1$ always holds.

Remark 1: The derivation of the model in (3) can also be illustrated similarly with the method in [18], which describes the process as Markov chain and results in a $N$-intertwined model.

The interpretation of the differential equations (3) in the node-base SIRS model is as follows: a) The change of the probability of node $v_{i}$ being infected during a time interval $\mathrm{d} t$ consists of two parts, i.e. the influence of the infected neighbors when node $v_{i}$ is susceptible and the probability of recovering when infected; b) The change of the probability of node $v_{i}$ being recovered during time interval $\mathrm{d} t$ is also made of two parts, i.e. the probability of recovering when node $v_{i}$ is infected and the probability of being susceptible when recovered.

Another typical practical example for the investigated model, other than the disease spreading case, is a marketing campaign. Assuming that a firm aims to sell more goods to a group of people. Then the propagation of the product can be described by the SIRS model. The people can be in one of three states, potential customer (susceptible), willing to buy (infected), ignoring all the related information of the product (recovered). The transmission process which is an interaction of neighboring nodes is explained as the fact that the arbitrary potential customer may turn to be willing to buy the product because of the influence by social neighbors who are willing to buy the product, e.g. word of mouth.

\section{AnAlysis AND CONTROL}

In this section, the equilibria of node-based SIRS model will be studied as well as the stability conditions.

To start with, the following matrix form of the model in (3) can be obtained by defining $A=\operatorname{diag}\left\{\alpha_{1}, \alpha_{2}, \ldots, \alpha_{N}\right\}$, $B=\operatorname{diag}\left\{\beta_{1}, \beta_{2}, \ldots, \beta_{N}\right\}$ and $\Gamma=\operatorname{diag}\left\{\gamma_{1}, \gamma_{2}, \ldots, \gamma_{N}\right\}$

$$
\begin{aligned}
& \dot{p}_{I}(t)=\left(I-P_{I}(t)-P_{R}(t)\right) W A p_{I}(t)-B p_{I}(t), \\
& \dot{p}_{R}(t)=B p_{I}(t)-\Gamma p_{R}(t),
\end{aligned}
$$

where $p_{I}(t)=\left[p_{1 I}(t), p_{2 I}(t), \ldots, p_{N I}(t)\right]^{T}$ and $P_{I}(t)=$ $\operatorname{diag}\left\{p_{1 I}(t), p_{2 I}(t), \ldots, p_{N I}(t)\right\}$ are the vector and diagonal matrix whose entries are the probabilities of all the nodes being infected at instance $t$, and $p_{R}(t)$ and $P_{R}(t)$ are defined equivalently for the recovered states. Hence, denoting $p(t)=$ $\left[p_{I}^{T}, p_{R}^{T}\right]^{T}$ and $\mathcal{P}(t)=-\left(P_{I}(t)+P_{R}(t)\right) W A$, the overall model can be written as

$$
\dot{p}(t)=\left[\begin{array}{ll}
W A-B & 0 \\
B & -\Gamma
\end{array}\right] p(t)+\left[\begin{array}{l}
\mathcal{P}(t) p_{I}(t) \\
0
\end{array}\right] .
$$

Then we can have the following results.

\section{A. Equilibria}

The equilibria of the node-based SIRS model on heterogeneous networks can be obtained by setting $\dot{p}_{i I}=0$ and $\dot{p}_{i R}=0$. Thus we have

$$
p_{i I}^{*}=\frac{\bar{\alpha}_{i}^{*}}{\left(1+\delta_{i}\right) \bar{\alpha}_{i}^{*}+\beta_{i}}, \quad p_{i R}^{*}=\frac{\delta_{i} \bar{\alpha}_{i}^{*}}{\left(1+\delta_{i}\right) \bar{\alpha}_{i}^{*}+\beta_{i}},
$$

where $\bar{\alpha}_{i}^{*}=\sum_{j=1}^{N} \alpha_{j} \omega_{i j} p_{j I}^{*}, \delta_{i}=\beta_{i} / \gamma_{i}$. Fruther analysis of (6) shows that the reasonable equilibria exist because

1) $p_{i I}^{*} \in[0,1)$ and $p_{i R}^{*} \in[0,1)$, which means they are proper probabilities.

2) $\left(p_{i I}^{*}+p_{i R}^{*}\right) \in[0,1)$, which infers the existence of $p_{i S}^{*}$, hence

$$
p_{i S}^{*}=\frac{\beta_{i}}{\left(1+\delta_{i}\right) \bar{\alpha}_{i}^{*}+\beta_{i}} .
$$

The origin is one of the equilibria of the node-based SIRS model in (5). According to the expression of $\bar{\alpha}_{i}^{*}$ in (2), the origin, i.e. $p_{i I}^{*}=p_{i R}^{*}=0$, is an equilibrium.

\section{B. Stability of the Origin}

The stability of the origin is crucial in disease control since the disease vanishes when all the people are in state $S$. The following theorem provides the condition to have an exponentially stable origin.

Theorem 1: The node-based SIRS model in (5) is globally exponentially stable, if the following matrix inequality holds

$$
\left[\begin{array}{ll}
A^{\frac{1}{2}} W A^{\frac{1}{2}}-B & \frac{1}{2} B \\
\frac{1}{2} B & -\Gamma
\end{array}\right]<0 .
$$

Proof: Introduce a Lyapunov function $V=\frac{1}{2} p^{T} p$ and we can obtain the time-derivative

$$
\begin{aligned}
\dot{V} & =p^{T} \dot{p} \\
& =p^{T}\left[\begin{array}{ll}
W A-B & 0 \\
B & -\Gamma
\end{array}\right] p+p^{T}\left[\begin{array}{l}
\mathcal{P} p_{I} \\
0
\end{array}\right] \\
& \leq p^{T}\left[\begin{array}{ll}
W A-B & 0 \\
B & -\Gamma
\end{array}\right] p .
\end{aligned}
$$

The inequality is correct because $p_{I}^{T} \mathcal{P} p_{I} \leq 0$ and the equality holds only when $p_{I}=0$. Hence,

$$
\begin{aligned}
\dot{V} \leq & p_{I}^{T}(W A-B) p_{I}+p_{R}^{T} B p_{I}-p_{R}^{T} \Gamma p_{R} \\
= & p_{I}^{T}(W A-B) p_{I}+\frac{1}{2} p_{I}^{T} B p_{R} \\
& +\frac{1}{2} p_{R}^{T} B p_{I}-p_{R}^{T} \Gamma p_{R} .
\end{aligned}
$$

Since $A$ is a diagonal matrix, we can rewrite $(W A-B)$ as $\left(A^{\frac{1}{2}} W A^{\frac{1}{2}}-B\right)$. it is obvious that if (8) holds, $\dot{V}<0$, which means that the model in (5) is globally asymptotically stable. Denote the matrix in (8) as $\mathcal{A}$. Since $\dot{V} \leq p^{T} \lambda_{\max }\{\mathcal{A}\} p=$ $2 \lambda_{\max }\{\mathcal{A}\} V$, the system is globally exponentially asymptotically stable. 


\section{Control Design}

We focus on the control problem of regulating the probability of infected and recovered to the desired values $p_{I}^{*}$ and $p_{R}^{*}$ via dynamically choosing recovering rates, i.e. replacing $\beta_{i}$ with the control input $u_{i}(t)$, of each node. Note that $u_{i}(t) \geq 0$ since recovering rate is non-negative. A practical way to imply this control strategy is to vaccinate people such that they can recover quickly. Although the desired value of the probability of a person being infected is usually 0 when aiming at impeding the disease spreading, the desired value differs for other applications. For instance, in marketing, $p_{i I}^{*}$, which can represent the desired possibility of individual $i$ to buy the product, is chosen close to 1 .

To tackle this control issue, $e_{I}(t)=p_{I}(t)-p_{I}^{*}$ and $e_{R}(t)=$ $p_{R}(t)-p_{R}^{*}$ are introduced as error variable vectors. Thus the system in (4) can be written as

$$
\begin{aligned}
\dot{e}_{I}(t) & =[\Xi(t)-U(t)]\left(e_{I}(t)+p_{I}^{*}\right), \\
\dot{e}_{R}(t) & =U(t) e_{I}(t)-\Gamma e_{R}(t)+U p_{I}^{*}-\Gamma p_{R}^{*},
\end{aligned}
$$

where $\Xi(t)=\left(I-E_{I}(t)-E_{R}(t)-P_{I}^{*}-P_{R}^{*}\right) W A$. $E_{I}(t)=\operatorname{diag}\left\{e_{1 I}, e_{2 I}, \ldots, e_{N I}\right\}$ and $E_{R}(t)=$ $\operatorname{diag}\left\{e_{1 R}, e_{2 R}, \ldots, e_{N R}\right\}$ are the diagonal matrices of the errors whose entries are the same with those of $e_{I}(t)$ and $e_{R}(t) ; U(t)=\operatorname{diag}\left\{u_{1}(t), u_{2}(t), \ldots, u_{N}(t)\right\}$ is the diagonal matrix of control inputs. The problem of designing the control strategy can be transformed to find dynamic $U(t)$ such that the origin of the closed-loop system in (11) is stable. Thus we obtain the following theorem as a result for this problem.

Theorem 2: The closed-loop node-based SIRS model on heterogeneous networks in (11) is globally asymptotically stable if $\lambda_{\max }\{\Xi(t)-U(t)\}<0$ for every time instance $t$.

Proof: First we prove the stability in a special case, i.e. the local stability. Rewrite the system in (11) as

$$
\begin{aligned}
& \dot{e}_{I}(t)=f\left(e_{I}(t), e_{R}(t)\right), \\
& \dot{e}_{R}(t)=g\left(e_{I}(t), e_{R}(t)\right) .
\end{aligned}
$$

Note that $f$ and $g$ are continuously differentiable. Then the Jacobian of the system in (12) can be obtained as

$$
J=\left[\begin{array}{ll}
\frac{\partial f}{\partial e_{I}} & \frac{\partial f}{\partial e_{R}} \\
\frac{\partial g}{\partial e_{I}} & \frac{\partial g}{\partial e_{R}}
\end{array}\right]=\left[\begin{array}{ll}
J_{1} & J_{2} \\
U(t) & -\Gamma
\end{array}\right]
$$

where

$$
\begin{aligned}
& J_{1}(i, j)= \begin{cases}-\sum_{k=1}^{N} \alpha_{k} \omega_{i k}\left(e_{k I}(t)+p_{k I}^{*}\right)-u_{i}(t), & i=j, \\
\left(1-e_{i I}-e_{i R}-p_{i I}^{*}-p_{i R}^{*}\right) \alpha_{j} \omega_{i j}, \quad i \neq j,\end{cases} \\
& J_{2}(i, j)=\left\{\begin{array}{l}
-\sum_{k=1}^{N} \alpha_{k} \omega_{i k}\left(e_{k I}(t)+p_{k I}^{*}\right), \quad i=j, \\
0, \quad i \neq j .
\end{array}\right.
\end{aligned}
$$

Recalling (2) and defining $\mathscr{A}=\operatorname{diag}\left\{\bar{\alpha}_{1}^{*}, \bar{\alpha}_{2}^{*}, \ldots, \bar{\alpha}_{N}^{*}\right\}$, we have the Jacobian in the origin

$$
\begin{aligned}
J(0)= & {\left[\begin{array}{ll}
\left(I-P_{I}^{*}-P_{R}^{*}\right) W A-U-\mathscr{A} & -\mathscr{A} \\
U & -\Gamma
\end{array}\right] } \\
= & {\left[\begin{array}{ll}
\left(I-P_{I}^{*}-P_{R}^{*}\right) W A-U & 0 \\
U & -\frac{1}{2} \Gamma
\end{array}\right] } \\
& +\left[\begin{array}{ll}
-\mathscr{A} & -\mathscr{A} \\
0 & -\frac{1}{2} \Gamma
\end{array}\right] .
\end{aligned}
$$

The Jacobian is Hurwitz when $\left[\left(I-P_{I}^{*}-P_{R}^{*}\right) W A-U\right]$ is Hurwitz, because $J(0)$ can then be expressed as the sum of two Hurwitz matrices. Note that the result is equal to $\lambda_{\max }\{\Xi(t)-U(t)\}<0$ when $e_{I}=0, e_{R}=0$. Thus the system in (11) is locally asymptotically stable [19].

As for the global asymptotic stability, because $U(t)$ is the control input diagonal matrix that we design, we can choose proper $U(t)$ such that $(\Xi(t)-U(t))$ is Hurwitz, i.e. $\lambda_{\max }\{\Xi(t)-U(t)\}<0$. Since $f$ and $g$ are both continuously differentiable, it follows that $J$ is always Hurwitz, which leads to the global asymptotic stability of the origin in (11)

Remark 2: By similarity transformation with $\left\{A^{\frac{1}{2}}, A^{-\frac{1}{2}}\right\}$, $(\Xi-U)$ can be turned into a symmetric matrix, which manifests that the eigenvalues of $(\Xi-U)$ are all real numbers and the condition given in Theorem 2 can be simply presented as $\lambda_{\max }\{\Xi(t)-U(t)\}<0$.

Theorem 2 only provides a theoretic method to find a control input, which is not good enough for practical use, because the eigenvalues should be calculated for unknown $U(t)$. Note that the entries of $(\Xi(t)-U(t))$ are the same as those of $J_{1}(t)$. While calculating the eigenvalue, the equation

$$
\operatorname{det}(\lambda I-(\Xi(t)-U(t)))=0 .
$$

is generally of $N$ th order. From the application point of view, one has to solve the above equation in every sampling instance and execute the trial and error process to check the negativeness of the largest eigenvalue, which requires a large amount of computation capacity. To tackle this problem, a more applicable algorithm is necessary, which is given in the following proposition.

Proposition 1: The system in (11) is globally asymptotically stable with the control input

$$
u_{i}(t)=\lambda_{\max }\{\Xi(t)\}+\epsilon_{i}, \quad i=1,2, \ldots, N .
$$

where $\epsilon_{i}$ is an arbitrarily small positive real number.

Proof: For any non-zero vector $x \in \mathbb{R}^{N}$, if the expression in (13) holds, we obtain

$$
x^{T}(\Xi(t)-U(t)) x<x^{T} \Xi(t) x-\lambda_{\max }\{\Xi(t)\} x^{T} x \leq 0,
$$

where the second inequality invariably holds according to the property of Rayleigh quotient. Thus the condition in Theorem 2 is fulfilled and we complete the proof.

Note that the knowledge of the entire states of the nodes are required by the controller, which is a maybe conservative but reasonable assumption for a controller from outside of the system. Taking disease spreading for example, the doctors and researchers who try to control the disease are able to 
know the situations of each potential patient. In marketing, as another instance, the information of potential customers can be obtained by questionnaires or analysing online comments.

\section{NUMERICAL EXAMPLES}

A random undirected connected graph with $N=20$ nodes is generalized for the simulation to demonstrate the results in this paper. The average degree of the graph is $\langle k\rangle=2.3$ with the degree span from 1 to 4 . The heterogeneity of transferring rates is achieved by randomly selecting within different regions. The ranges of the parameters of each simulation are listed in Table I.

TABLE I

THE RANGES OF PARAMETERS

\begin{tabular}{|c||c||c||c|}
\hline & $\alpha_{i}$ & $\beta_{i}$ & $\gamma_{i}$ \\
\hline Figure 2 & $(0.05,0.15)$ & $(0.25,0.35)$ & $(0.3,0.4)$ \\
\hline Figure 3 & $(0.1,0.2)$ & $(0.6,0.8)$ & $(0.3,0.5)$ \\
\hline Figure 4(a) \& 5(a) & $(0.45,0.55)$ & $(0.85,0.95)$ & $(0.4,0.5)$ \\
\hline Figure 4(b) \& 5(b) & $(0.45,0.55)$ & $u_{i}$ & $(0.4,0.5)$ \\
\hline
\end{tabular}

Firstly, we examine the approximation made in the expression in (1). The residual sums of squares (RSS) of $p_{I}$ and $p_{R}$ are chosen as the criterion. In detail, the following system is considered.

$$
\begin{aligned}
\dot{\tilde{p}}_{i I}(t)= & \left(1-\tilde{p}_{i I}(t)-\tilde{p}_{i R}(t)\right)\left(1-\prod_{j \in \mathcal{N}\left(v_{i}\right)}\left(1-\alpha_{j} \tilde{p}_{j I}\right)\right) \\
& -\beta_{i} \tilde{p}_{i I}(t), \\
\dot{\tilde{p}}_{i R}(t)= & \beta_{i} \tilde{p}_{i I}(t)-\gamma_{i} \tilde{p}_{i R} .
\end{aligned}
$$

By comparing the system in (14) with the system in (3), we define the RSS of $p_{I}$ and $p_{R}$ as $\left\|p_{I}-\tilde{p}_{I}\right\|_{2}^{2}$ and $\left\|p_{R}-\tilde{p}_{R}\right\|_{2}^{2}$, respectively. As is manifested in Figure 2, the influence of the replacement towards system variables, $p_{I}$ and $p_{R}$, converges, which demonstrates the effectiveness of our approach.
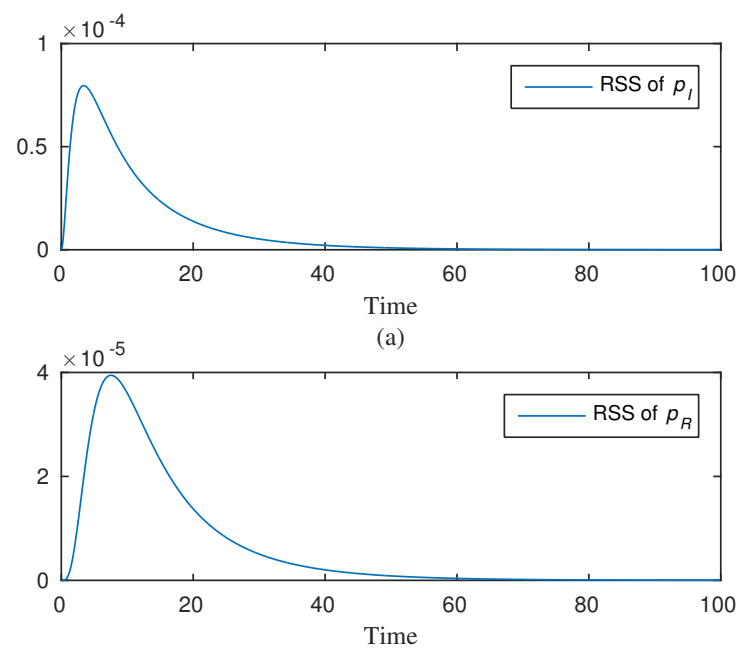

(b)

Fig. 2. Estimation errors of $p_{I}$ and $p_{R}$ for neglecting higher order terms in (1)
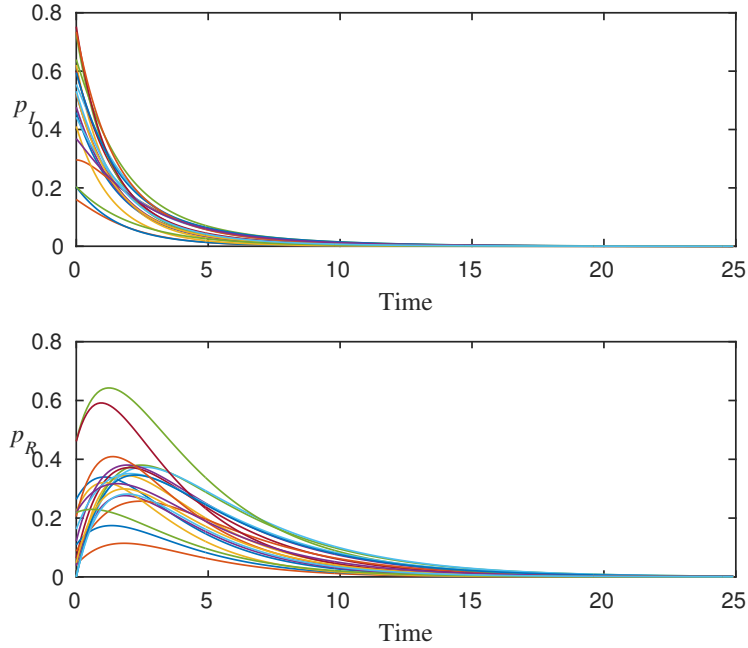

Fig. 3. Global exponential stability of origin of the SIRS model in a random graph with 20 nodes

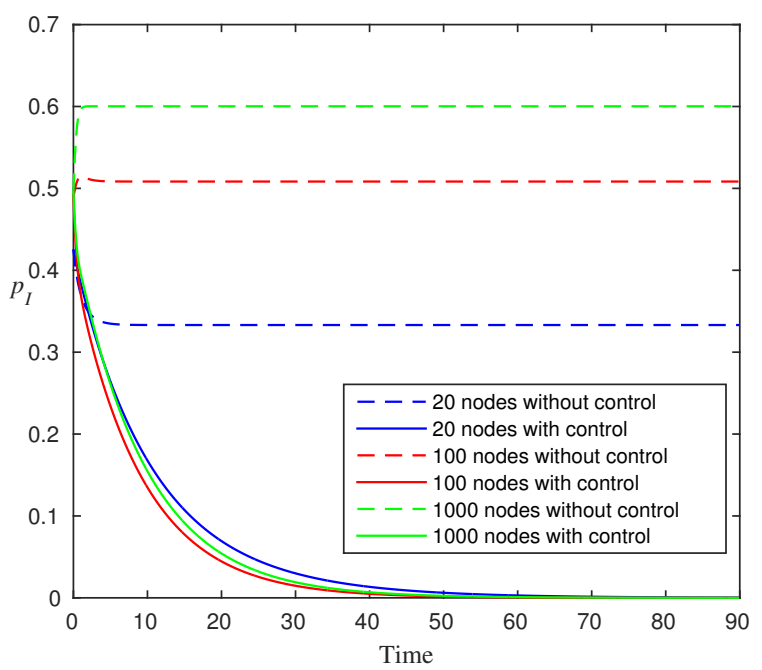

Fig. 4. (a) Probabilities of being infected in graphs with 20,100 and 1000 nodes with and without control

Secondly the global stability of origin is presented in Figure 3. The initial conditions, i.e. $p_{I}(0)$ and $p_{R}(0)$, are chosen randomly such that $p_{i I}(0), p_{i R}(0),\left(p_{i I}+p_{i R}\right) \in$ $[0,1]$. When the condition given in Theorem 1 is fulfilled, the probabilities of being infected and recovered converge to 0 within finite time.

Finally, for the control design, the desired probabilities of infected and recovered are both set to 0. Considering the influence of the complexity of the graph, we take the graphs with 20,100 and 1000 nodes as examples. For contrast, both the graph structure and the parameters $\left(\alpha_{i}\right.$ and $\left.\gamma_{i}\right)$ are the same for the graphs with the same number of nodes. We can see that under the same initial conditions, $p_{I}$ and $p_{R}$ can be regulated to target value under control (solid lines in Figure 45 ) but fail to reach the desired value when the system is under no control (dash lines in Figure 4 and Figure 5). 


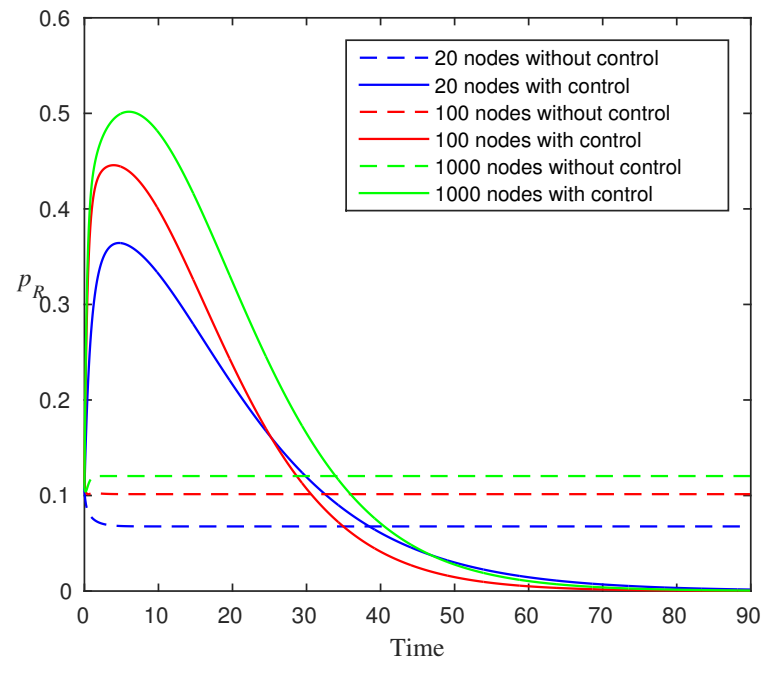

Fig. 5. Probabilities of being recovered in graphs with 20, 100 and 1000 nodes with and without control

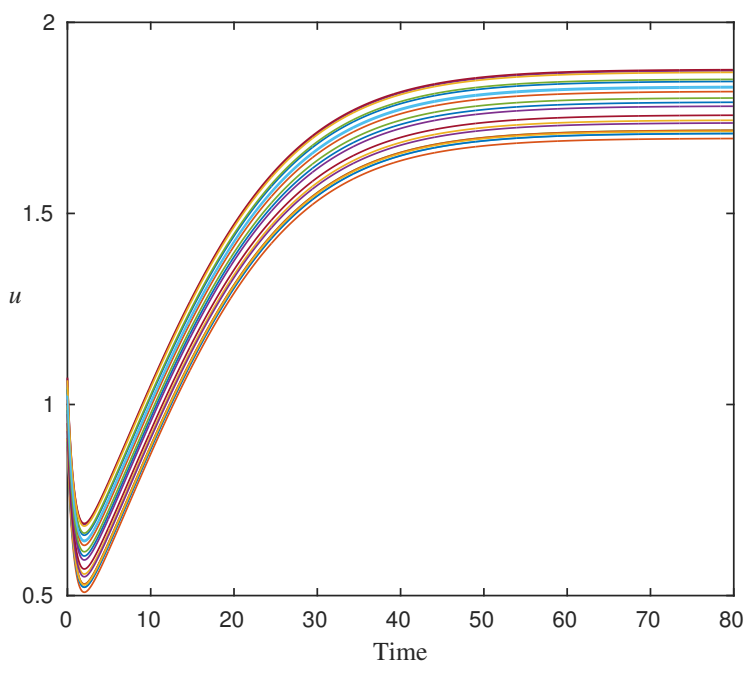

Fig. 6. Control input of each node in a 20-node graph

There is no doubt that as the number of nodes increases, the simulation is more time-consuming because the maximum eigenvalue of a square matrix of larger dimension needs to be computed. Also note that the sudden overshoots in Figure 5 come from the increase of the recovering rates, which leads to the infected more likely to turn to be recovered. The control input presented in Figure 6, taking the graph with 20 nodes for instance, is calculated according to the method given in Proposition 1 with the arbitrary parameter $\epsilon_{i}$ randomly chosen in the region $(0,0.2)$.

\section{CONCLUSION}

The node-base SIRS model on heterogeneous networks that describes the interactions between the nodes is proposed.
The existence of the equilibria has been proved via analysing the steady-state of the model. Taking origin as a special case, the condition for its global exponential stability has been obtained, which is also the condition for disease free case. Considering the control problem of reaching desired states, a controller to dynamically generate recovering rates is designed via analysing the Jacobian.

Future work will focus on the study of the stability of non-zero equilibria and optimal controller design. Reducing the number of controlled nodes and the information needed for controller design will also be inspected.

\section{REFERENCES}

[1] Y.-F. Chou, H.-H. Huang, and R.-G. Cheng, "Modeling information dissemination in generalized social networks," IEEE Communications Letters, vol. 17, no. 7, pp. 1356-1359, 2013.

[2] Y. Lin, J. C. Lui, K. Jung, and S. Lim, "Modeling multi-state diffusion process in complex networks: theory and applications," in 21st European Conference on Artificial Intelligence, Prage, Czech, 2014.

[3] V. Isham, S. Harden, and M. Nekovee, "Stochastic epidemics and rumours on finite random networks," Physica A: Statistical Mechanics and its Applications, vol. 389, no. 3, pp. 561-576, 2010.

[4] Y. Hayel, S. Trajanovski, E. Altman, and H. W. hand P. V. Mieghem, "Complete game theoretic characterization of sis epidemics protection strategies," in IEEE 53rd Annual Conference on Decision and Control (CDC), Dec. 2014.

[5] J. Liu, Y. Tang, and Z. Yang, "The spread of disease with birth and death on networks," Journal of Statistical Mechanics: Theory and Experiment, vol. P08008, 2004.

[6] D. Watts and S. Strogatz, "Collective dynamics of small world networks," Nature, vol. 393, pp. 440-442, 1998.

[7] T. Schelling, Micromotives and Macrobehavior. New York: W. W. Norton \& Company, 1978.

[8] J. Goldenberg, "Talk of the network: A complex systems look at the underlying process of word-of-mouth," Marketing Letters, vol. 12, no. 3, pp. 211-223, 2001.

[9] C. Castellano and R. Pastor-Satorras, "Thresholds for epidemic spreading in networks," Phys. Rev. Lett, vol. 105, no. 21, pp. 87-101, 2010.

[10] A. Khanafer, T. Basar, and B. Gharesifard, "Stability properties of infected networks with low curing rates," in American Control Conference, Portland, OR, American, 2014, pp. 3579-3584.

[11] J. Kephart and S. White, "Directed-graph epidemiological models of computer viruses," in 1991 IEEE Computer Society Symposium on Research in Security and Privacy, Oakland, CA, USA, 1991.

[12] K. Kandhway and J. Kuri, "Campaigning in heterogeneous social networks: optimal control of si information epidemics," IEEE/ACM Transactions on Networking, 2014.

[13] A. Barrat, M. Barthelemy, and A. Vespignani, Dynamical Processes on Complex Networks. Cambridge: Cambridge University Press, 2012.

[14] Y. Hu, L. Min, and Y. Kuang, "Modeling the dynamics of epidemic spreading on homogenous and heterogeneous networks," Applicable Analysis, vol. 94, no. 11, pp. 2308-2330, 2015.

[15] L. Chen and J. Sun, "Global stability and optimal control of an sirs epidemic model on heterogeneous networks," Physica A:Statistical Mechanics and its Applications, vol. 410, pp. 196-204, 2015.

[16] M. Draief and L. Massouli, Epidemics and Rumours in Complex Networks. Cambridge: Cambridge University Press, 2010.

[17] Q. Huang, L. Min, and X. Chen, "Susceptible-infected-recovered models with natural birth and death on complex networks," Mathematical Methods in the Applied Sciences, vol. 38, no. 1, pp. 37-50, 2015.

[18] P. V. Mieghem, J. Omic, and R. Kooij, "Virus spread in networks," IEEE/ACM Transactions on Networking, vol. 17, no. 1, pp. 1-14, 2009.

[19] H. K. Khalil, Nonlinear Systems. Prentice Hall, 2002. 